\title{
Uncertainty on Virtualization and Internet of Things (IOT): The Connectivity problem and the rise of localize edge computing
}

\author{
Sharipah Setapa ${ }^{1}$, Shahrol Hisham Baharom ${ }^{1}$, Luke Jing Yuan ${ }^{1}$ \\ Advance Computing Lab, Mimos Berhad, Kuala Lumpur, Malaysia \\ *Correspondence: sharipah, shahrol.baharom,jyluke\}@ mimos.my
}

\begin{abstract}
The connectivity failure could be contributed by device, intermediate device, configuration, problem of duplicates IP, software problem or physical cable. The rise of connectivity problem has become a trend with a virtual interconnectivity when involves Internet of Things (IoT). Many connectivity are dependent to each other. A dependency between each interconnection can cause a limited information transfer if the chain is broken which causes inaccurate data path during travelling to the destination. Application sensors will collect a huge data, but the consumption of the architecture's loading and processing based on real time and traffic can cause a segmentation of suggestion to change. One of the example is a community based driven application, which depends on users to activate the application to get an information in real time. The more users activate the application, the more accurate route suggestion will be. This paper is about a study of connectivity, data challenge, opportunities prediction and analysis flow from application sensor based on a dataset obtained through a simulated community driven navigation.
\end{abstract}

Keywords: Huge data, data challenge, connectivity, prediction, session design

\section{Introduction}

When Internet was introduced it changed the way how people communicate remotely with each other. It gives alternative communication means to traditional methods such as conventional telephone and postal mail. Mobile applications via Internet which support text, video, audio and images spontaneously such as skype or WhatsApp, create a new era of communication. The diverse applications in a mobilized device increase things around us to be interconnected.

A connectivity has actually happened in every single step of user activity. The growing of internet of things (IoT) can cause increasing demand to interconnect all the devices to a respective network. Connectivity is increased when using a device such as smartphone to control the things remotely. One of the examples is to control house appliances by remotely monitoring situation such as light, video surveillance and refrigerator.

The thing is real and physical, but when it comes to the processing and provision of data from the things it will involve different users and technologies. There are cloud computing and virtualization which support a similar pool of resources to variety of users ${ }^{[1]}$. The connectivity which involves cloud for processing the data is to coordinate the data which needs a storage, information access, reliability and security ${ }^{[2]}$.

Cloud computing and virtualization have become complimentary for IoT to become enhanced and up to date (S.
Nastic et.al 2014) F. Li et.al, 2013) ${ }^{[3][4]}$. With a huge number of things being interconnected, there is a need for a service which can be offered by cloud computing. If one-point connectivity is down, then the backup resources can be automatically be shared.

There are different causes which make network inaccessible. In the case of a virtual network, if it is using VirtualBox, the network can be broken down into different categories for of interconnectivity. This is to understand the basic of how the connectivity depends on the network attachment to make the connectivity run as intended.

Table 1: Virtual Network Classification in virtualbox ${ }^{[5]}$

\begin{tabular}{ll}
\hline $\begin{array}{l}\text { Network } \\
\text { attachment }\end{array}$ & $\begin{array}{l}\text { Connectivity } \\
\text { A virtual machine to internet and } \\
\text { vice versa }\end{array}$ \\
NAT & $\begin{array}{l}\text { Connect virtual machine to each } \\
\text { Nether as well as to the Internet }\end{array}$ \\
Internal & $\begin{array}{l}\text { Virtual machine to virtual } \\
\text { network }\end{array}$ \\
machine & $\begin{array}{l}\text { Virtual machine to virtual } \\
\text { Host-only } \\
\text { Adapter }\end{array}$ \\
\hline
\end{tabular}




\begin{tabular}{ll}
\hline & Virtual machine to host, Virtual \\
Bridged & machine to Virtual machine, \\
Adapter & $\begin{array}{l}\text { Virtual machine to internet and } \\
\text { vice versa }\end{array}$ \\
\hline
\end{tabular}

Each connectivity represents different permission to access the network. This means if an IoT device needs to access different network, it needs different permission or configuration to allow the distribution of data to the end user or device for a better connectivity ${ }^{[6]}$.

A device such as smartphone can be converted to a sensor. Each step of the smartphone connectivity represents different network communication as shown in figure 1. During the simulation and the calculation of the data, the processing is depending on the service offered by the cloud.

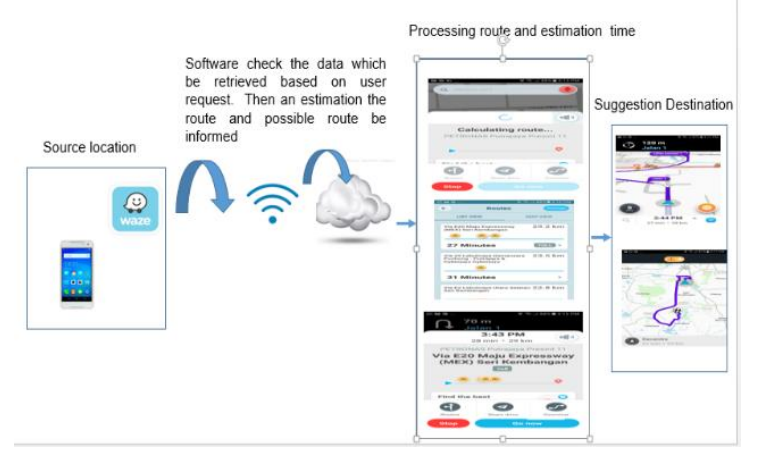

Figure 1: Connectivity through virtualization and cloud Every activity resides in each stage is to complement each other. Each stage becomes connected to generate a connectivity to deliver an estimation of the route and the best route. Track and trace is visible during activity to get all the information from various things during the connectivity from source to destination. If a congestion happens then the information will be passed to the source for situation analysis before deciding any action. A list of routes will be given and the best route suggestion is based on the current situation on that day. This collected information prevent the source from taking the road or highway which can increase a traffic flow. The rest of this paper is organized as follows: Section II discusses the related works, which are relevant and providing background knowledge to our work. Section III discusses the problem statement and objectives of our proposed work. Section IV discusses the methodological concept for our case study case. Section V discusses the interconnectivity influence and a general challenge faced. Section VI gives an explanation about a navigation application criteria. Section VII discusses the real-time simulation setup and simulation for measuring data results and finally, concludes the proposed study and explores the feasibility of future work.

\section{Literature Review}

Cloud computing technologies have been intensively exploited in the development and management of the largescale IoT systems ${ }^{[3][7][8][9]}$. Merlino et.al ${ }^{[10]}$ describes how network virtualization is a key enabler of infrastructureoriented IoT solutions. It proposes cloud-based network virtualization for IoT. Daniel E. O'leary ${ }^{[11]}$ examines the relationship between 'Big Data', 'Internet of Things' and internet sign. A thing can generate a wide range of measurements that can be used to provide a sign or output that indicates some event or to show that a place is busy, for example a sensor can availability of parking slot at any particular moment. Rudyar Cort'es et.al ${ }^{[12]}$ studied a dataset from a popular IoT sport tracking application which involved a big data. It provides and analyses the data flow. Mayra Samaniego et.al ${ }^{[13]}$ proposed an implementation of dew servers to provision virtual resources towards specific groups of edge devices. They integrated smart virtual resources at the edge level to provide IoT services and data views directly from edge devices with block chain for security. R. Cortés et.al ${ }^{[14]}$ mentioned that the consolidated power might not be efficient to handle the velocity of data flow produced by sensors.

\section{Problem Statement and Objective}

The connectivity stages in IoT application cannot be viewed and are dependent on each other to provide an information. In a community driven application, the connectivity needs to learn based on the input from the community to make the path suggestion become accurate. A challenge inherent from the community driven application was investigated in depth to know how number of communities are interconnected according to time and how this situation can influence the suggested path based on time duration.

\section{Methodology}

In order to acquire real traces for our IoT connectivity, a specific navigation route was simulated. A simulation was done through some application using a smartphone as a sensor. A GPS was enabled to trace a user or vehicle location and all activities around the route between source and destination. A summary output will present a statistic which was assessed during the specific session to get a full life cycle of the data measurement. This statistic was generated along the route with a measurement of the data to identify the challenge and potential of data behavior for future benefit.

\section{Interconnectivy Challenge}

An application sensor provides a report based on reporting by users and the number of reports it receives. The report is kept in a different virtualization database to be updated in certain period. This is to closely monitor the relevant information and 
to get a manual report to be used and adjusted for different scenario prediction.

Communication connectivity is possible through $\mathrm{Wi}-\mathrm{Fi}$, physical connection or sensor. With smartphone the connectivity is at our fingertip and contribute to our daily life activity. A potential possibility can be suggested during an interconnectivity in community navigation driven application i.e. on the estimation of traveler's arrival. However, the information or suggestion changes depending on the time it is produced.

When the connectivity is down, a backup can be accessed through other alternative connectivity supported by the cloud. Although the cloud represents a robust and reliable architecture for IoT computation offloading, the thing itself needs more power during collection of data. More power is needed to offload data from things for processing. For example, a smartphone assessing a community application will drain power from its battery.

\section{Application Community Driven Navigatioanl}

Our study case focuses on one of community's driven applications which is navigation service. The service is currently tracking all activities along transportation roads. Waze is a popular navigator application. It allows users to track the route, destination, share their route activities and publish reports or any activity in the community for everyone to see.

There are challenges faced during simulation of the destination route for vehicular which can be categorized as:

a. How to collect and observe the data during simulation of the route

The problem is on how to capture with accurate time because each result will change dynamically when defining source and destination

b. Data arrangement and classification.

How to arrange and classify parameter data which will be collected and how to declare the period of changing between source and destination

\section{c. Data Representation for viewing}

How to represent the data based on factor which impacts to route.

d. How to interpret the data result

How to analyse and interpret the result which will be retrieved.
The application which runs on smartphones and tablet, enables GPS to track or obtain a position about location and route detail with real-time input from users. The input provides information on events happening along the road. Through this application any activity on the road can be monitored for us to manage the destination accordingly.

An observation shows that there is exist a structure of sequence during a mobility journey to a specific destination which involves virtualization layer. A simulation data was passed to a virtual machine to be stored and kept for further references or as a guidance as shown in figure 4 .

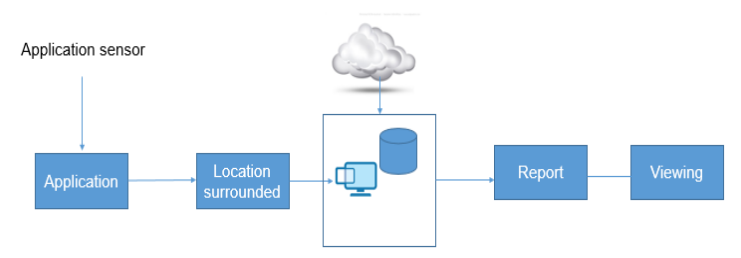

Figure 2: Level surrounded based application information

However, there was an intermittent in each stage of the interaction between application, surrounded location and cloud itself.

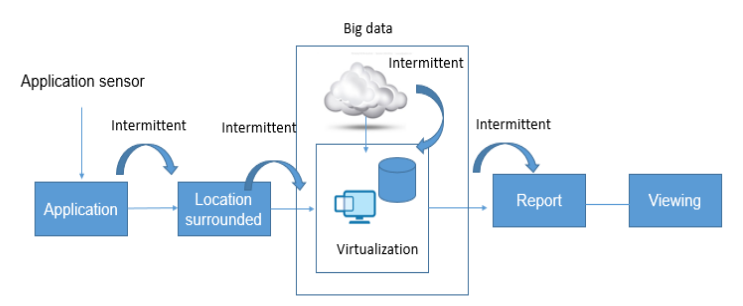

Figure 3: Level surrounded and intermittent

\section{Simulation For Measuring the Data}

In order to show the progress of tracking for our testing, a sample dataset was collected. This dataset is to show the progress of users' activities reported within twenty four hours by the same source and destination in urban areas but within a different time period. The period session was divided into afternoon, morning and the next afternoon with a possibility of a traffic jam during a peak hour.

Table 2: Type of data arrangement

\begin{tabular}{lll}
\hline Data arrangement & user & $\begin{array}{l}\text { User } \\
\text { report }\end{array}$ \\
Cluster1_afternoon & 28128 & 718 \\
Cluster2_afternoon & 28231 & 721 \\
Cluster3_afternoon & 28629 & 741 \\
Cluster4_morning & 31209 & 826 \\
Cluster5_morning & 30708 & 397 \\
\hline
\end{tabular}




\begin{tabular}{lll}
\hline Cluster6_morning & 28545 & 74 \\
Cluster7_afternoon & 31048 & 68 \\
Cluster 8_afternoon & 31851 & 723 \\
Cluster 9_afternoon & 32833 & 818 \\
\hline
\end{tabular}

A few datasets were collected to get a statistic on the progress within a specific and fixed location which provide a report on surrounding users before being stored in a database. The morning session is from 9.30 am to 10.30 am whereas afternoon session is between $4 \mathrm{pm}$ to $5 \mathrm{pm}$. The approach of scaling the data is to get a good analysis e.g. to show an increasing or decreasing behaviour.

A duplicate session was conducted the next day to complete the cycle of twenty-four hours. The collected data showed that there was an increase in reports and users during the second afternoon

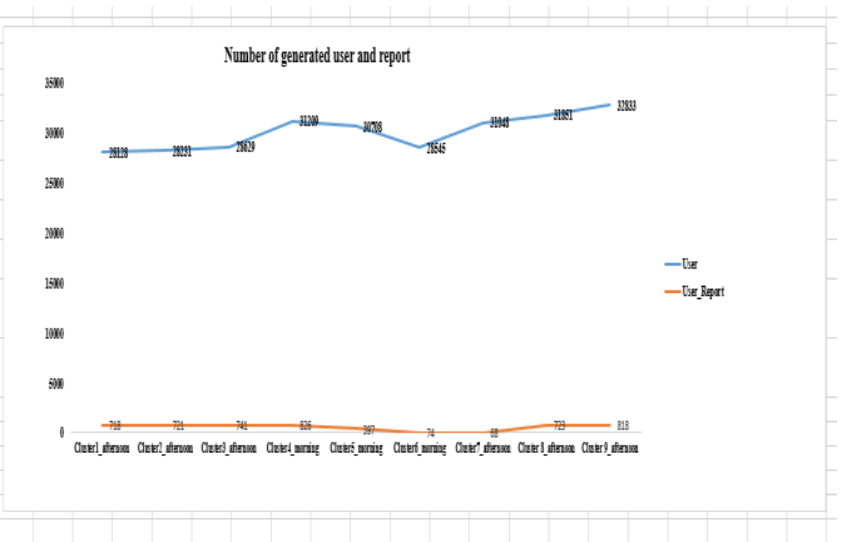

Figure 4: Number of generated user and report

The data was simulated by giving a small number for further analysis as shown in figure 5 .

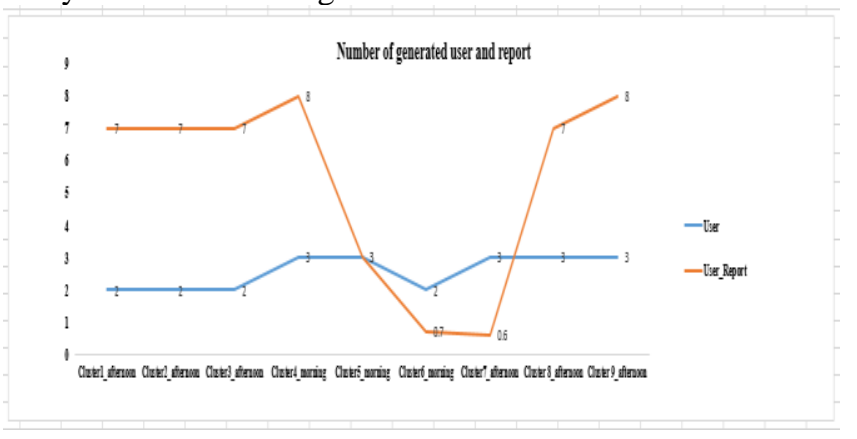

Figure 5: Number of generated user and report by scaling into small number

Figure 5 shows that at the end of the afternoon session the number of users is decreasing while the report is increasing. It can be predicted that traffic is not busy both after the end of the morning and early afternoon. The increase and decrease fluctuate according to the interval time which was arranged as shown in figure 5. This data was being accumulated for appropriate next scaling. It is was loaded into a database for viewing the timeline sequence session and to analyse whether the selected session needed to be changed accordingly.

The input workload of the user is continuous but the user's rate varies over time section. A key challenge is to design and implement a scalable way to support a number of connected users in order to handle peaks of user reports.

When the scaling was multiplied by two, the value is bigger for the next day as shown in figure 6. However, this value is a prediction for us to take a precaution to estimate how to handle the traffic based on ad hoc situation in a specific urban area. As an example, if there is an event to celebrate on the next day in the urban area, the scaling can be multiplied with different scale numbers to predict possible situation so that any respective precaution can be taken.

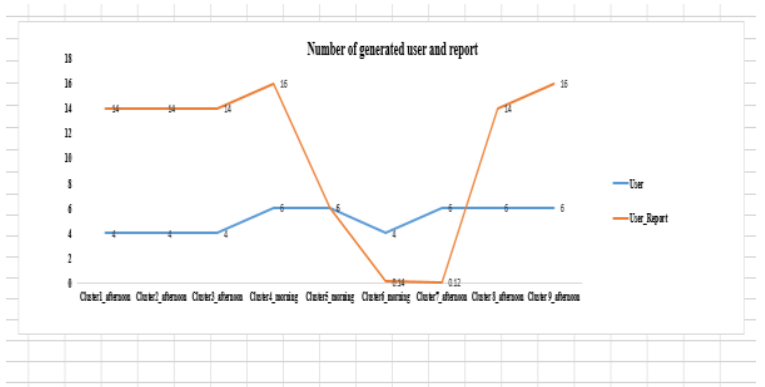

Figure 6: Number of generated user and report by multiplying the scaling

A sensing-as-a-service can be utilized based on mobility through smartphone. The service is being studied primarily to get a general overview of the surrounding gates before a report can be viewed.

There are a few categories of services which replicate cloud functionalities, even though they have different interrelation for interconnecting node within IoT. Below are those services.

\section{A Network as a service}

This service provides a network which can capture data at the edge devices to be transported to another node of IoT device which requires the same data.

\section{B Application as a service}

In each edge node, an application can be shared with other node, for example in a case if the data in a camera needs to be transferred to a smartphone using an application which is compatible with each other.

\section{Mobility as a service}

For the transportation like a car, each application was mobilized when IoT devices are collecting route estimation. 


\section{Location as a service}

Location of the dynamically running need a GPS to trace the current location for comparison along the surrounding activities.

\section{F. Database and viewing as a service}

This database can be used as a reference to show how the gap happens between current and previous session. This will provide a study on what parameter is involved and which parameter will cause different situation when compared to the current.

\section{Conclusion}

This discussion is about the challenges that exist for interconnecting tracking and opportunities prediction which can be retrieved during tracking of data flows of community driven application. The simulation data depends on how cloud can serve the IoT in a better way. A continuous simulation with long period without a comprehensive cloud and enhanced virtualization will potentially influence the data because there are a many services to be entertained in the cloud. Existing cloud that is reacting to thing will contribute to the development for the stabilization of IoT and prediction especially for intelligence computing and analytic.

If a smart traffic urban city needs to be implemented, the dependency to user to activate the application is a problem due to battery drain while mobilizing. However a study of previously obtained data can provide a logical value for traffic control of urban city. At the same time the valuable data can be utilized for user safety, for example when using grab car or any pool car. It suggests that any of the things which is located along an urban area would be able to provide information based on preferences of users. The preference is linked remotely to the things, whether a static or mobile. Each thing becomes a sensor to deliver any data and it is not depending on existing GPS to track during its movement. This is possible if the initialization of the preference also acknowledges the things which exist between source and destination.

\section{References}

[1]. Merlino, Giovanni \& Bruneo, Dario \& Longo, Francesco \& Distefano, Salvatore \& Puliafito, Antonio. (2015). Cloud-Based Network Virtualization: An IoT Use Case. 199-210. 10.1007/978-3-319-25067-0_16.

[2]. Lin Gu, Deze Zeng and Song Guo Vehicular cloud computing: A survey 2013 IEEE Globecom Workshops, GC Wkshps 2013

[3]. S. Nastic, S. Sehic, D. Le, H. Truong and S. Dustdar, "Provisioning Software-Defined IoT Cloud
Systems," 2014 International Conference on Future Internet of Things and Cloud, Barcelona, 2014, pp. 288-295, doi: 10.1109/FiCloud.2014.52.

[4]. F. Li, M. Vögler, M. Claeßens, and S. Dustdar, "Efficient and Scalable IoT Service Delivery on Cloud.," IEEE CLOUD, pp. 740-747, 2013.

[5]. Student, "Enable VirtualBox VM to VM Communications on Windows / Ubuntu 16.04 | 18.04", https://websiteforstudents.com/enablevirtualbox-vm-to-vm-communications-onwindows-ubuntu-16-04-18-04/' Website for Students, 2018.

[6]. F. Bonomi, R. Milito, J. Zhu, and S. Addepalli, "Fog Computing and Its Role in the Internet of Things," Proc. first Ed. MCC Work. Mob.cloud Comput., pp. 13-16, 2012.

[7]. M. M. Hassan, B. Song, and E.-N. Huh. A framework of sensor-cloud integration opportunities and challenges. In ICUIMC, 2009.

[8]. J. Soldatos, M. Serrano, and M. Hauswirth. Convergence of utility computing with the internetof-things. In IMIS, pages 874-879, 2012.

[9]. M. Yuriyama and T. Kushida. Sensor-cloud infrastructure-physical sensor management with virtualized sensors on cloud computing. In NBiS, 2010.

[10]. Merlino, Giovanni \& Bruneo, Dario \& Longo, Francesco \& Distefano, Salvatore \& Puliafito, Antonio. (2015). Cloud-Based Network Virtualization: An IoT Use Case. 199-210. 10.1007/978-3-319-25067-0_16.

[11]. Daniel E. O'Leary*. (2013) 'Big Data', The 'Internet of Things' and The 'Internet of Signs', Intelligent Systems in Accounting, Finance and Management. Intell. Sys. Acc. Fin. Mgmt. 20, 53-65 (2013).Published online in Wiley Online Library (wileyonlinelibrary.com) DOI: 10.1002/isaf.1336

[12]. Rudyar Cortes., Xavier Bonnaire, Olivier Marin \& Pierre Sens. (2015). Stream processing of healthcare sensor data: studying user traces to identify challenges from a big data perspective. Procedia Computer Science 52 (1), volume number (1004 1009l), pages.

[13]. M. Samaniego, C. Espana and R. Deters Smart Virtualization for IoT. 2018 IEEE International Conference on Smart Cloud (SmartCloud), New York, NY, 2018, pp. 125-128, doi:10.1109/SmartCloud.2018.00028.

[14]. R. Cortés, X. Bonnaire, O. Marin, and P. Sens, "Stream Processing of Healthcare Sensor Data: Studying User Traces to Identify Challenges from a Big Data Perspective," Procedia Comput. Sci., vol. 52, pp.1004-1009, 2015. 